\title{
Preparation of Total Flavonoids Tablets of Portulaca Oleracea L
}

\author{
Zhao Rui*, Hou Jin Zuo, Zhang Yu Yan
}

Heilongjiang Bayi Agricultural University, it is located in Daqing City, Heilongjiang Province, China

DOI: $10.36347 /$ sjet.2020.v08i04.002

| Received: 12.02.2020 | Accepted: 22.02.2020 | Published: 11.04.2020

*Corresponding author: Zhao Rui

Abstract

Original Research Article

In this study, total flavonoids of portulaca oleracea were extracted by ultrasonic method and prepared as tablets, single factor test was used to screen the optimal preparation process of tablets. Results show that, when the pressure was 7.5, the adhesive was $4 \%$ cross-linked povidone dry powder, and the excipient formula was lactose: dextrin 1:1, all the tablets obtained were in accordance with the indicators under the tablet of Chinese pharmacopoeia.

Keywords: Portulaca flavone tablets; content determination; adhesive.

Copyright @ 2020: This is an open-access article distributed under the terms of the Creative Commons Attribution license which permits unrestricted use, distribution, and reproduction in any medium for non-commercial use (NonCommercial, or CC-BY-NC) provided the original author and source are credited.

\section{INTRODUCTION}

Portulaca oleracea L. is a kind of wild plant with the same origin of medicine and food. It contains a variety of medicinal components, which are mainly used in treating diarrhea, regulating blood glucose and detoxifying decrease internal heat in clinical practice. Its main components include polysaccharides, flavonoids and alkaloids [1]. Among them, the content of total flavonoids accounts for $7.67 \%$ of the total weight of purslane, which has a variety of medicinal activities [2]. As a kind of medicinal and edible homologous plant, its potential great use value, in order to make it more convenient to play a variety of value, the researchers have conducted a variety of studies on its natural pharmaceutical preparations. Currently, the dosage forms have been reported as: portulaca cinnamomum granules [3], portulaca oleracea compound oral liquid, portulaca oleracea polysaccharide granules [4] and portulaca oleracea polysaccharide injection. However, no natural pharmaceutical preparation of portulaca oleracea flavonoids has been reported. Therefore, in this experiment, portulaca oleracea flavones were prepared as functional food with tablet as carrier, laying a foundation for the research on the development of modern dosage forms of flavonoids.

\section{MATERIALS AND METHODS \\ Chemical and Reagents}

Rutin standard (purity $\geq 98 \%$ ) was purchased from baicaoyuan biotechnology Co., Ltd. (Jiangxi, China). Povidone K30 was purchased from sanlandchem International Inc. (USA). Lactose, dextrin, microcrystalline cellulose, starch and magnesium stearate are all biological agent provided by aubostar biotechnology Co., Ltd. (Beijing, China). Crosslinked polyvidone xl-10 purchased from Shanghai changwei pharmaceutical accessories technology Co., Ltd. (Shanghai, China). All other reagents are of analytical grade purchased from Liaoning quanrui reagent Co., Ltd. (liaoning, China).

\section{Preparation of Flavonoids from Portulaca Oleracea} $\mathbf{L}$

Dried purslane, crushed into evenly sized segments. Petroleum ether was used as degreasing solvent, the ratio of material to liquid was $1: 10$, and ultrasonic degreasing occurs twice at $40^{\circ} \mathrm{C}$, each time for $0.5 \mathrm{~h}$. Filter and ventilate purslane until completely dry. Set aside. $60 \%$ ethanol was used as the extraction solvent, the ratio of material to liquid was 1:30, and it was sealed and soaked overnight. Ultrasonic extraction was conducted at $50^{\circ} \mathrm{C}$ for 2 times, each time for 1 hour. Filtration, collection of filtrate, concentration of 10 times volume, extraction of chloroform, ethyl acetate, n-butanol, to obtain concentrated solution of oleracea flavones. Freeze overnight at $-80^{\circ} \mathrm{C}$ and freeze dry for $48 \mathrm{~h}$ to obtain the dry powder of oleracea flavones.

\section{Preparation of Flavonoids Tablets of Purslane}

Because the tablets have the characteristics of small volume, high bioavailability, low requirements for storage conditions and stable quality, this experiment used the single-factor test method to prepare portulaca oleracea flavones as tablets, and screened the prescription process according to the quality standards 
of tablets in the 2015 edition of Chinese pharmacopoeia.

\section{Tablet Preparation Process}

The raw materials and auxiliary materials were mixed evenly at a ratio of $1: 1.5$, then $6 \%$ cross-linked povidone was added in internal addition, 95\% ethanol was wetted, and adhesive was added to prepare soft materials. Granulation was made by 12 -mesh sieve, dried at $55^{\circ} \mathrm{C}$ for $30 \mathrm{~min}$, and the grains were integrated by no. 1 sieve and no. 2 sieve, and 5\% magnesium stearate was mixed evenly and immediately pressed into pieces [5].

\section{Screening Pressure}

Preparation pressure is one of the basic factors influencing the quality standard of traditional Chinese medicine tablets. Too much stress leads to slow disintegration; Too little pressure, produce loose pieces. Therefore, in order to ensure the quality of tablets, film pressure screening. Lactose was used as a single excipient, and the tablet appearance, weight variations, hardness and disintegration time were taken as indicators [6] to determine the optimal pressure.

\section{Screening Filler}

According to the properties of the flavonoids of purslane, lactose, dextrin, starch and microcrystalline cellulose (MCC) with low moisture absorption and good compressibility were selected as fillers, and the better fillers were screened by taking granulation status, difference in tablet weight, hardness, yield and disintegration time as investigation indexes.

\section{Screening Adhesive}

Because of the poor adhesion of the dry powder of portulaca oleracea, which affects the particle formation rate, the different adhesives were screened. The optimum adhesives were selected based on the conditions of granulation, difference in weight, hardness, yield and disintegration time.

\section{Determine the Excipient Formula}

Based on the above factors, the optimal excipient formula was determined.

\section{Determination of Flavonoids in Tablets of Purslane}

The content of total flavonoids in flavonoids tablets was determined by the content determination method of common tablet materials in 2015 edition of Chinese pharmacopoeia. Sodium nitrite colorimetry was used to prepare rutin standard solution, and the absorbance value was measured at $475 \mathrm{~nm}$. The standard curve was established to calculate the content of flavonoids and the relative percentage $\mathrm{K}$ in the tablet.

The calculation formula is as follows:

$$
\mathrm{K}(\%)=\left(\mathrm{C} \times \mathrm{V}_{1} \times \mathrm{V}_{2}\right) / \mathrm{M} \times \mathrm{V}_{3} \times 100 \text { [7] }
$$

Where, $\mathrm{K}$ is the relative percentage $(\%)$ of flavonoid raw materials in flavonoid tablets; $\mathrm{C}$ is the sample concentration $(\mathrm{mg} / \mathrm{mL}) ; \mathrm{V}_{1}$ is the volume $(\mathrm{mL})$ of the tablet aqueous solution; $\mathrm{V}_{2}$ is the fixed volume $(\mathrm{mL})$ of the chromogenic reaction; $\mathrm{M}$ is the weight of flavonoids $(\mathrm{mg})$ of purslane; $\mathrm{V}_{3}$ is the volume $(\mathrm{mL})$ used for color reaction.

\section{RESULT}

Screening results of preparation pressure of tablets.

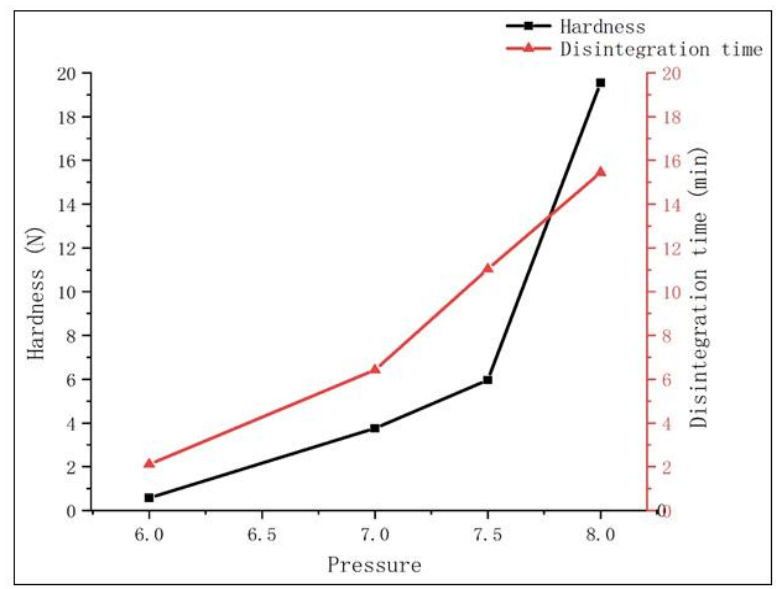

Picture-1: Test results of hardness and disintegration time of film pressure

In this study, the preparation pressures of tablets were $6,7,7.5$ and 8 . According to the general rule 0101 of the 2015 edition of Chinese pharmacopoeia, the appearance, weight difference, hardness and disintegration time of the tablets were tested. The results are shown in Picture-1 and Table-1. When the pressure is 7.5 , all indicators are in accordance with the provisions of the national pharmacopoeia.

Table-1: Tablet weight difference and tablet appearance test results of tablet preparation pressure $(\mathrm{n}=\mathbf{2 0})$

\begin{tabular}{|l|l|l|}
\hline Pressure & Weight difference & Tablet appearance \\
\hline 6 & Disqualified & Powdery tablet, complete, uniform, matte \\
\hline 7 & Qualified & Micronized tablet, complete, uniform and slightly shiny \\
\hline 7.5 & Qualified & Glossy tablets, complete and smooth \\
\hline 8 & Qualified & Glossy tablets, complete and smooth \\
\hline
\end{tabular}

\section{Screening Results of Tablet Filler}

Lactose, dextrin, microcrystalline cellulose and starch were selected as fillers in the experiment. The results of various indicators were shown in Picture $2 \&$
3 and Table-2. The results showed that all the indexes of lactose and dextrin were in conformity with the standards of Chinese pharmacopoeia. 


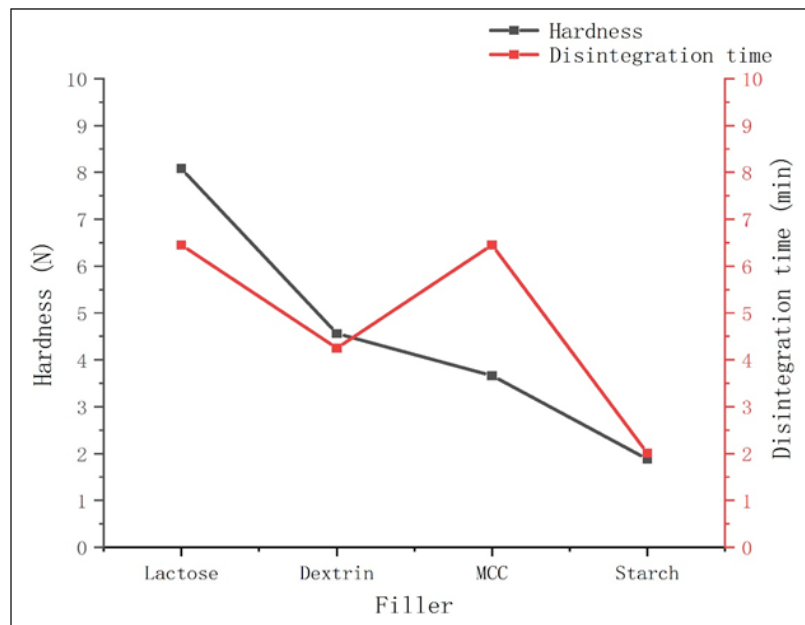

Picture-2: Test results of hardness and disintegration time of filler

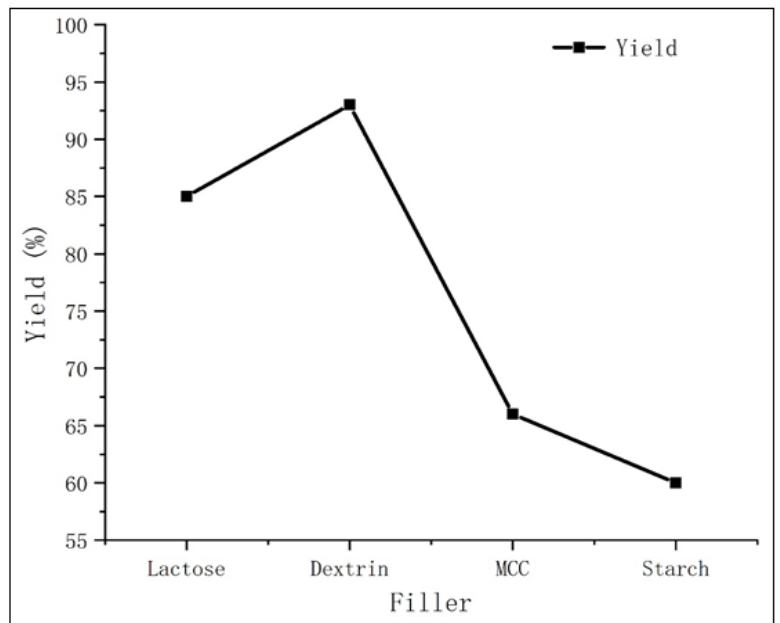

Picture-3: Filler yield test results

Table-2: Tablet weight difference of filler and granulation appearance test results $(n=20)$

\begin{tabular}{|l|l|l|}
\hline Filler & Weight difference & Tablet appearance \\
\hline Lactose & Qualified & The particles are easy to form, soft material too sticky, less powder \\
\hline Dextrin & Qualified & Particles easy to form, adhesion, small powder, one-sided spot \\
\hline MCC & Qualified & Particles are not easy to shape, size is not uniform, too much fine powder \\
\hline Starch & Disqualified & Particles are not easy to form, too much fine powder, tablets soft \\
\hline
\end{tabular}

\section{Screening results of tablet adhesives}

Adhesives with different viscosity were selected in the experiment. The viscosity was successively dextrin pulp, glucose syrup, sucrose syrup, polyvidone (PVP) dry powder and polyvidone solution.
The test results of various indicators are shown in picture $4 \& 5$, and Table- 3 . The results showed that the PVP powder was in accordance with the pharmacopoeia, and was more suitable as a binder for the flavonoids tablets of purslane.

Table-3: Adhesive weight difference and tablet appearance test results $(n=20)$

\begin{tabular}{|l|l|l|}
\hline Adhesive & Weight difference & Tablet appearance \\
\hline Dextrin pulp & Qualified & Powder tablets, particles easy to shape, one-sided smooth \\
\hline Glucose syrup & Qualified & Powder tablets, particles easy to shape, sticky \\
\hline Sucrose syrup & Qualified & Powder tablets, granules are not easy to shape and granulate \\
\hline PVP dry powder & Qualified & Powder tablets, particles easy to shape, one-sided luster \\
\hline PVP solution & Qualified & Glossy tablets, smooth, slightly variegated, not easy to granulate \\
\hline
\end{tabular}

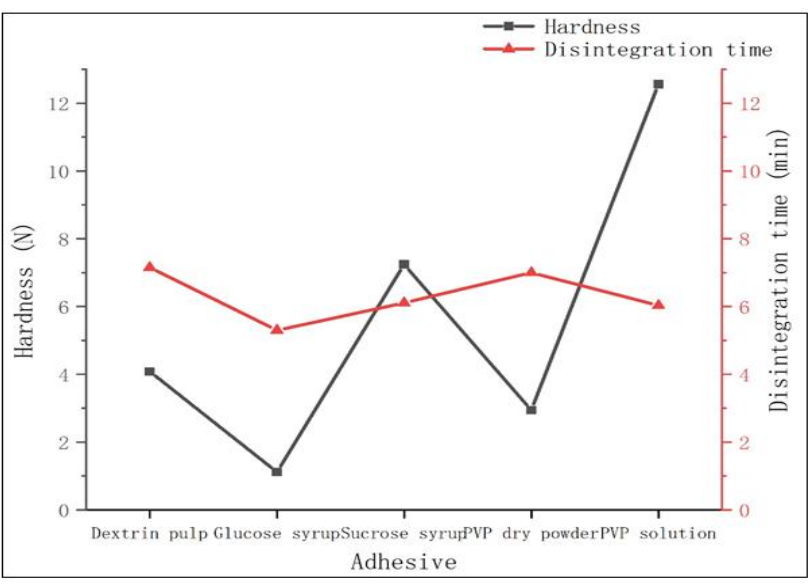

Picture-4: Test results of hardness and disintegration time of adhesive

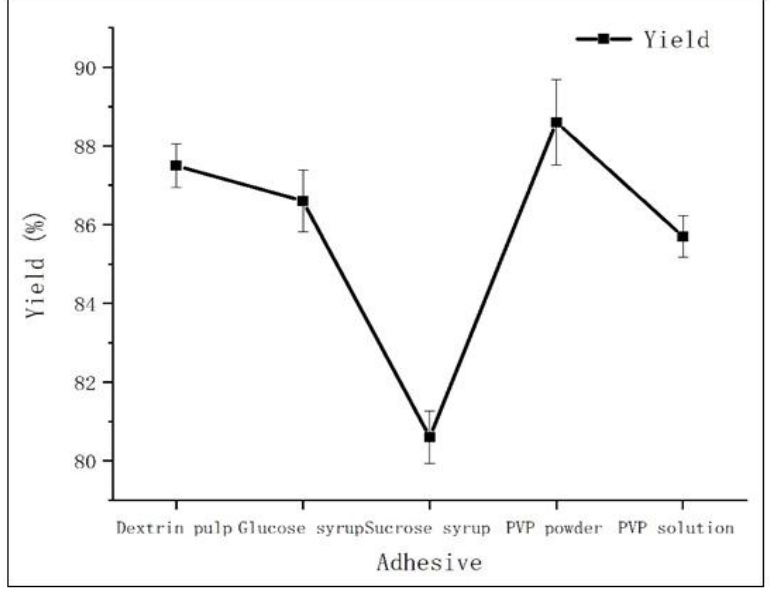

Picture-5: Test result of molding rate of adhesive 


\section{Screening Results of Tablet Formulation}

The results showed that the single lactose excipient had some phenomena, such as over adhesion of soft material, difficulty in granulation and low molding rate. Accordingly, match different component formula, detect each index. Dextrin can enhance the adhesion of raw materials and improve the tablet forming rate. MCC can enhance the hardness of tablets

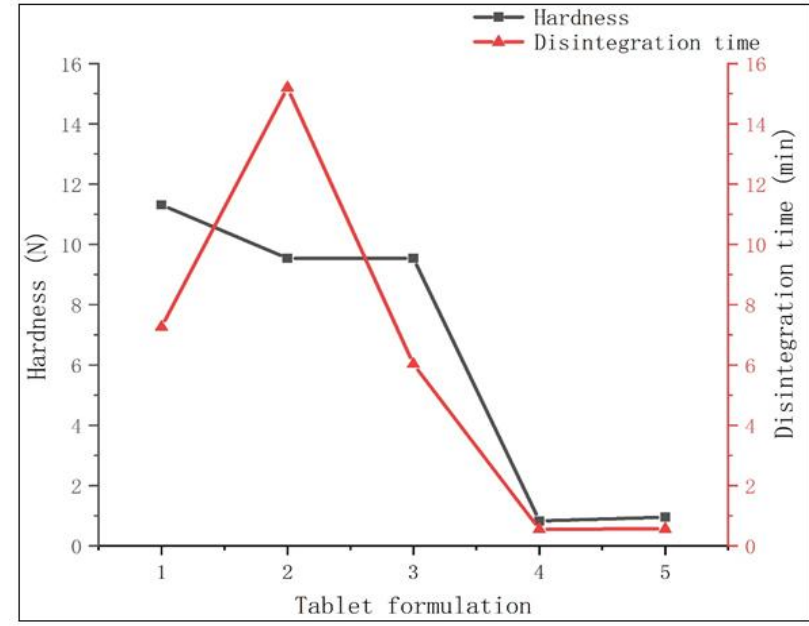

Picture-6: Test results of hardness and disintegration time of tablet formulation and the viscosity of neutralizing adhesives, and starch can alleviate the phenomenon that soft materials are not easy to be granulated due to the excessive adhesiveness of soft materials. The results are shown in Picture $6 \& 7$ and Table-4. Picture-8, Table-4, 1 is lactose: dextrin $=1: 1 ; 2$ is lactose: dextrin $=1: 2 ; 3$ is lactose: dextrin=2:1; 4 is lactose: $\mathrm{MCC}=2: 1 ; 5$ is lactose: $\operatorname{starch}=2: 1$.

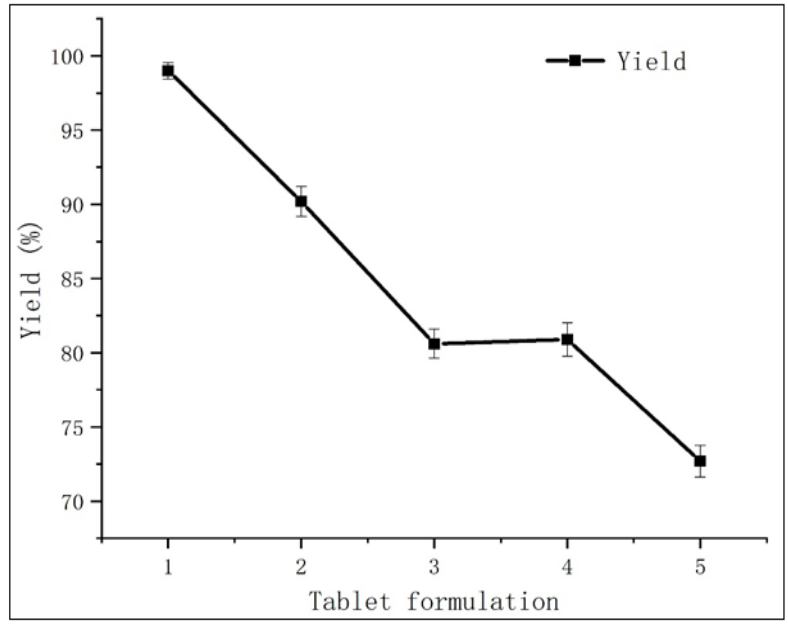

Picture-7: Yield test results of tablet formulation

Table-4: Tablet formulation of the tablet weight difference and tablet appearance test results $(\mathrm{n}=20)$

\begin{tabular}{|l|l|l|l|}
\hline $\begin{array}{l}\text { Tablet } \\
\text { formulation }\end{array}$ & $\begin{array}{l}\text { Weight } \\
\text { difference }\end{array}$ & Tablet appearance & $\begin{array}{l}\text { Comprehensive } \\
\text { score }\end{array}$ \\
\hline 1 & Qualified & Lustrous tablets, particles easy to form, incomplete and complete smooth & 98.9 \\
\hline 2 & Qualified & Glossy tablets, particles easy to shape, soft materials micro-adhesive & 90.2 \\
\hline 3 & Qualified & Powder tablets, particles are easier to shape, soft materials too sticky & 77.9 \\
\hline 4 & Qualified & Powder tablets, particles are not easy to form, too much fine powder & 66.7 \\
\hline 5 & Disqualified & Powder tablets, particles are not easy to form, too much fine powder & 60.3 \\
\hline
\end{tabular}

\section{Determination of tablet content}

The rutin standard curve measured at $475 \mathrm{~nm}$ is shown in Picture-8.

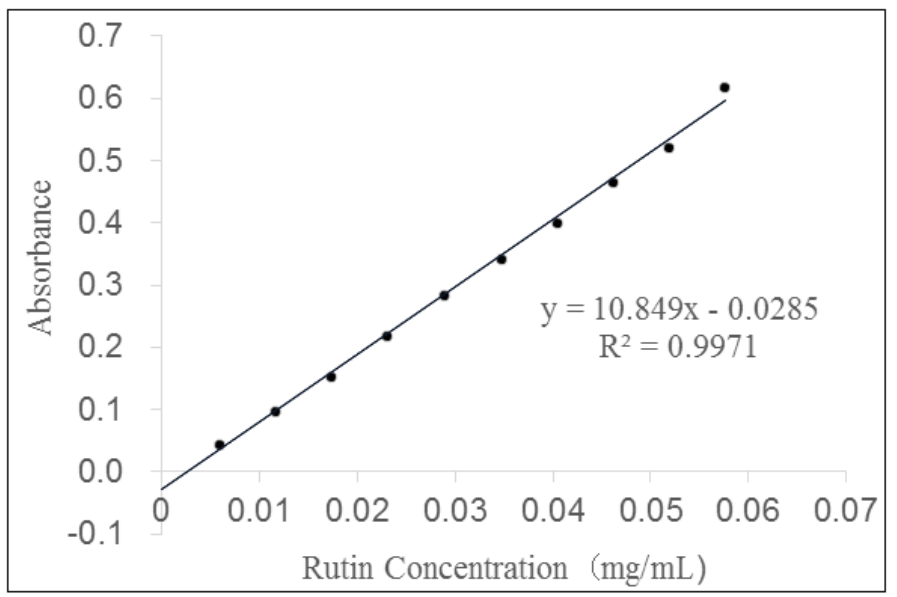

Picture-8: Rutin standard curve 
Table-5: Determination results of flavonoids tablets of purslane $(n=3)$

\begin{tabular}{|l|l|l|l|l|}
\hline Tablet (mg) & A & $\mathbf{C}(\mathbf{m g} / \mathbf{m L})$ & Flavonoid M (mg) & Content (\%) \\
\hline 50 & 0.123 & 0.014 & 17.50 & $35.00 \%$ \\
\hline 100 & 0.275 & 0.028 & 35.00 & $35.00 \%$ \\
\hline 150 & 0.421 & 0.041 & 51.75 & $34.50 \%$ \\
\hline 200 & 0.548 & 0.053 & 66.48 & $33.24 \%$ \\
\hline 250 & 0.645 & 0.062 & 77.55 & $31.02 \%$ \\
\hline 300 & 0.811 & 0.077 & 96.72 & $32.24 \%$ \\
\hline
\end{tabular}

\section{DISCUSSION}

During the preparation of tablets, the moisture content affects the stability of drugs, so the wetinducing characteristics of raw materials should be considered when choosing the excipients. Moreover, the raw material of flavonoids of purslane is acidic drugs, which have poor pressability. Therefore, excipients that do not react with acidic drugs, but also have low moisture absorption, high viscosity and do not delay drug disintegration should be selected. When making ordinary tablets, commonly used fillers are lactose, sucrose, starch, dextrin, pre-gelatinized starch, microcrystalline cellulose, inorganic salt, etc [8]. Lactose has good mobility and compressibility, and there is no chemical reaction to most drugs, and the pressure of the pill is fine, beautiful and hard, and the release of the drug is faster, and the content of the main drug is less determined, and the time limit of the tablet is not prolonged [9]. Dextrin has strong viscosity and can be used in combination with ethanol to enhance the hardness of the tablet, but excessive dosage will lead to unilateral patina and slow disintegration and dissolution. At the same time, the dextrin is not soluble in cold water and ethanol, and if the content is determined, it is easy to influence the accuracy of the results. Sucrose is easily absorbent and is used for loose texture, but acidic and alkaline drugs can increase the effect of this line, so it is not suitable for the distribution. Microcrystalline cellulose is not soluble in water, ethanol and other organic solvents, soluble in alkali solution, can enhance the hardness, liquidity and disintegration of the tablet, and the amount of the tablet is better when the content is 30 percent. Starch is not soluble in cold water and ethanol, the property is very stable, hygroscopicity is small, but the compressibility is poor, often with dextrin, sugar powder with the use of tablets alone is too loose. To sum up, the use of lactose and microcrystalline cellulose alone, with the use of starch, dextrin, is more in line with the requirements of oleracea flavone tablet filler. Disintegrating agents commonly used in tablet pressing include cross-linked polyvidone, carboxymethyl cellulose and derivatives of carboxymethyl cellulose salt, etc [10], and the amount of disintegrating agent and the way of adding have a great impact on the disintegrating rate and dissolution rate. The amount of disintegrating agent is generally 5$20 \%$ of the total tablet weight. The higher the dosage, the faster the disintegration efficiency. In this paper, we only need to make the flavone tablets of purslane as ordinary tablets, so we only need to comply with the provisions of Chinese pharmacopoeia. Therefore, we choose to add disintegrating agent inside, and the dosage is $6 \%$ of the total tablets. Because most natural drug extracts have poor compressibility, the adhesive with different viscosity is very important to the production process. The viscosity of commonly used adhesives is starch paste $<$ dextrin paste $<$ syrup $<$ glue paste. In this paper, dextrin $(83 \mathrm{~g} / \mathrm{L})$, glucose syrup $(500$ $\mathrm{g} / \mathrm{L})$, sucrose syrup (500 g/L), povidone dry powder $(4 \%)$, povidone solution $(20 \mathrm{~g} / \mathrm{L})$ were investigated. Therefore, lactose, dextrin, microcrystalline cellulose and starch were selected as fillers, cross-linked polyvidone was used as disintegrating agent, and $4 \%$ polyvidone dry powder was used as adhesive to prepare the flavonoids tablets of purslane.

\section{CONCLUSION}

In this paper, the preparation pressure, type and ratio of fillers, and type of adhesives of tablets were investigated by single factor test, and the optimal process was determined according to the indexes of tablets in 2015 edition of Chinese pharmacopoeia. The results showed that the optimal process of oleracea flavone tablets was: the ratio of raw and excipient was $1: 1.5$, and the excipient was lactose: dextrin 1:1.Adding $6 \%$ crosslinked povidone, $4 \%$ povidone dry powder, $95 \%$ ethanol wetting to prepare soft. After granulation, the tablets were dried and whole, $0.05 \%$ magnesium stearate was added, and the pressure was 7.5 to prepare the tablets.

\section{REFERENCES}

1. Wang TN, Liu YT, Xiao FQ, Ju BW, Yan MM, Ye DD, Xu DM. Modern research on chemical constituents and pharmacological activities of Portulaca oleracea. Chinese Journal of Exiperimental Traditional Medical Formulae. 2018; 24(6):224-234.

2. Hou JZ, Zhao R, Liu YP, Jia GY. Portulaca oleracea L. flavone: A Review of Extract and Pharmacological Effects. Anhui Agricultural Science Bulletin. 2019; 25(01):30-33.

3. Ren LL, Tan ZS, Liang YN, Yu JG, Yin G, Liu L, Song ZX, Huang WJ, Wang Z. Preparation of Portulaca oleracea-Cinnamon Granules and Its Effect on Intestinal Flora in Mice with Ulcerative Colitis. Chinese Journal of Microecology. 2019; 31(2):130-137.

4. Xue ZM, Liu YP, Zhao R. Preparation of Portulaca Polysaccharide Granules. Anhui Agricultural Science Bulletin. 2018; 24(23):26-28. 
5. Yan JY. Extraction of Total Saponins from Stems and Leaves of American Ginseng and Preparation of Tablets. Shandong University of Traditional Chinese Medicine, Shandong China. 2017;22.

6. [6] Zhang HQ, Huang QY. Study on the Preparation of Licorice Effervescent Tablets. The Food Industry. 2018; 39(1):112-115.

7. Wang CX. Purification of Flavonoids from Portulaca oleracea and Its Anti-diabetic Activity. Jiangxi Science and Technology Normal University, JiangXi China. 2015;18-22.
8. Tang XY. Study on pharmacy of Ginkgo biloba tablets. Chengdu University of TCM, Cheng Du China. 2013;17-26.

9. Yang Y. Talking about the common thinner for tablets. The Asia-pacific Traditional Medicine. 2009; 5(08):163-164.

10. Meng XY, Wu SG, Jia CJ, Cai Y, Li ZK, Zhang RY. Application of high-efficiency tablet disintegrant in pharmaceutical tablets. Journal of Traditional Chinese Veterinary Medicine. 2002; (2):45-46. 